\title{
Vertraglich vereinbarter Sex gegen Entgelt ist nicht sittenwidrig
}

\author{
Anmerkungen zu BGE 147 IV 73
}

Die strafrechtliche Abteilung des Bundesgerichts weist die Beschwerde eines Studenten ab und bestätigt dessen Verurteilung wegen Betrugs. Der Beschwerdeführer hatte seiner Vertragspartnerin die vereinbarten CHF 2000 für die von ihr erbrachten sexuellen Dienstleistungen nicht bezahlt und stellte sich auf den Standpunkt, es sei kein gültiger Vertrag zwischen ihnen zustande gekommen. Das Bundesgericht folgt in seinen Erwägungen der wohl herrschenden Lehre, dem Bundesrat und gewissen kantonalen Urteilen, wonach der Sexarbeitsvertrag nicht (mehr) per se sittenwidrig sei, und lässt der sexuellen Dienstleistung einen Vermögenswert zukommen. Es bestehe kein Widerspruch zu den der Gesamtrechtsordnung immanenten ethischen Prinzipien und Wertmassstäben, womit die Vereinbarung als gültig zu qualifizieren sei. Damit ist ein lang erwartetes Urteil gefällt. Es bleiben weitere rechtliche Massnahmen zu treffen.

I. BGE 147 IV 73

1. Sachverhalt

2. Prozessgeschichte

3. Erwägungen des Bundesgerichts

II. Anmerkungen

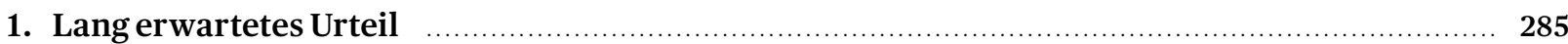

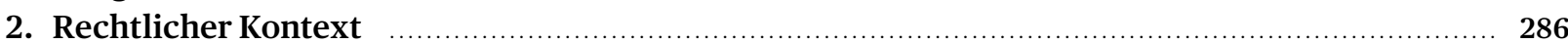

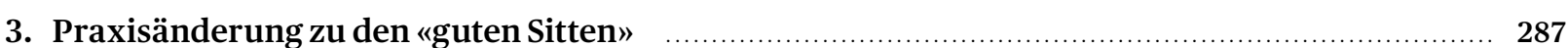

4. Weitere Massnahmen gegen Stigmatisierung und Diskriminierung bleiben nötig _................289

Zitiervorschlag:

SANDRA HOTZ / MERET LÜDI, Vertraglich vereinbarter Sex gegen Entgelt ist nicht sittenwidrig, sui generis 2021, S. 283 


\section{BGE 147 IV 73}

\section{Sachverhalt}

1 Dem Urteil des Bundesgerichts vom 8. Januar 2021 lag folgender Sachverhalt zugrunde: Ein Student (Beschwerdeführer) suchte unter einem falschen Namen mittels Inserat im Internet nach bezahlter Sexdienstleistung. Auf das Inserat meldete sich die Privatklägerin mit der Frage, was sie für die gebotenen CHF 2000 tun müsse. Der Beschwerdeführer antwortete ihr, er wolle eine Nacht mit ihr verbringen und Sex haben. Er versicherte ihr zudem mehrmals, er werde ihr die CHF 2000 nach der gemeinsamen Nacht bezahlen.

2 Die beiden verabredeten sich einige Wochen später an einem Bahnhof, wo der Beschwerdeführer die Privatklägerin mit seinem Personenwagen abholte. Im Auto versicherte er auf Nachfrage wahrheitswidrig, dass er das Geld bei sich trage und es ihr nach dem Geschlechtsverkehr geben werde. Die beiden fuhren in ein Hotel, wo sich die Privatklägerin nach dem Geschlechtsverkehr wiederum nach der Bezahlung erkundigte. Der Beschwerdeführer versicherte erneut, er werde sie nach einem weiteren Mal Geschlechtsverkehr bezahlen. Nach dem zweiten Mal Sex schlief die Privatklägerin ein. Der Beschwerdeführer löschte daraufhin alle Chat-Verläufe, E-Mails und Fotos von sich auf ihrem Mobiltelefon, entwendete ihr CHF 41 aus dem Portemonnaie und verliess das Hotelzimmer ohne Bezahlung der vereinbarten CHF 2000.

\section{Prozessgeschichte}

3 Das Kreisgericht St. Gallen verurteilte den Beschwerdeführer erstinstanzlich wegen Betrugs, Datenbeschädigung und geringfügigen Diebstahls zu einer bedingten Geldstrafe von 50 Tagessätzen zu je CHF 110 sowie einer Busse von CHF 300. Ausserdem verpflichtete es den Beschwerdeführer zur Leistung von Schadenersatz in der Höhe von CHF 2041 an die Privatklägerin. Die vom Beschwerdeführer dagegen erhobene Berufung wies das Kantonsgericht St. Gallen ab. Mittels Beschwerde in Strafsachen vor Bundesgericht beantragte der Beschwerdeführer den Freispruch von der Anklage wegen Betrugs sowie die vollumfängliche Abweisung der Zivilklage.

\section{Erwägungen des Bundesgerichts}

Das Bundesgericht prüft in seinem Urteil, ob der Tatbestand des Betruges gem. Art.146 Abs.1StGB2 von der Vorinstanz zu Recht als erfüllt angesehen wurde. Strittig ist

\footnotetext{
BGE 147 IV 73.

2 Schweizerisches Strafgesetzbuch vom 21. Dezember 1937 (StGB; SR311.0).
}

zum einen, ob eine arglistige Täuschung gegeben ist, und zum anderen, ob ein Vermögensschaden i. S. d. Bestimmung vorliegt.

Zunächst macht das Bundesgericht Ausführungen zur 5 Frage, ob das Tatbestandselement der arglistigen Täuschung erfüllt ist. Gemäss bundesgerichtlicher Rechtsprechung ist eine «Täuschung [...] eine unrichtige Erklärung über Tatsachen, die darauf gerichtet ist, bei einem andern eine von der Wirklichkeit abweichende Vorstellung hervorzurufen. [...] Als Tatsachen, über welche getäuscht werden kann, gelten auch innere Tatsachen, wie etwa Leistungswille und Erfüllungsbereitschaft» (E.3.1). Eine Täuschung könne sodann auch in einem konkludenten Verhalten liegen. So erkläre, "wer einen Vertrag eingeh[e], in der Regel konkludent die innere Tatsache, dass er gewillt [sei], die Leistung zu erbringen» (E.3.1). Eine Täuschung i. S. v. Art.146 Abs.1StGB muss ferner eine arglistige sein (E.3.2). Gemäss Rechtsprechung gelte die Vorspiegelung des Leistungswillens grundsätzlich als arglistig i. S. v. Art.146 StGB, da es sich beim Leistungswillen um eine innere Tatsache handle, welche durch die andere Vertragspartei nicht direkt überprüfbar sei. Es könne nur dann nicht von Arglist ausgegangen werden, wenn die Erfüllungsfähigkeit des Täuschenden mittels Nachforschungen überprüfbar sei und sich aus einer zumutbaren Prüfung ergeben würde, dass der Täuschende den Vertrag gar nicht erfüllen könne. Denn wem die Erfüllung gar nicht möglich sei, könne auch keinen ernsthaften Erfüllungswillen haben (E. 3.3).

Das Bundesgericht folgt den Ausführungen der Vorin- 6 stanz, wonach der Beschwerdeführer die Privatklägerin über seinen Zahlungswillen i. S. v. Art.146 Abs.1 StGB getäuscht habe. Angesichts des Verhaltens des Beschwerdeführers (teuer aussehender Personenwagen, wiederholt wahrheitswidrige Aussage, er sei im Finanzbereich tätig) habe die Privatklägerin von der Zahlungsfähigkeit des Beschwerdeführers ausgehen dürfen. Ebenso habe sie aufgrund seines Verhaltens keinen Anlass gehabt, an seinem Zahlungswillen zu zweifeln. Die Privatklägerin habe sich somit nicht leichtfertig verhalten. Das Bundesgericht kommt deshalb zum Schluss, dass die Täuschung durch den Beschwerdeführer als arglistig anzusehen sei (E. 4). Allerdings erwägt das Bundesgericht auch (E. 4.2), dass «mutmassliche Gepflogenheiten bei Sexdienstleistungsverträgen im professionellen Gewerbe nicht unbesehen auf den vorliegenden Fall übertragen werden können» (siehe dazu Rz. 24).

Nach der Bejahung der arglistigen Täuschung kommt 7 das Bundesgericht zur Frage, ob ein Vermögensschaden i. S. v. Art. 146 Abs. 2 StGB vorliegt. Hierzu hat es die Frage zu beantworten, ob ein Vertrag über die Erbringung einer sexuellen Dienstleistung gegen Entgelt als sittenwidrig 
zu betrachten sei. Bei einem sittenwidrigen Vertrag gehöre das vereinbarte Entgelt nicht zum strafrechtlich geschützten Vermögen und es könne folglich auch kein Vermögensschaden im Sinne der Bestimmung vorliegen (E. 6). Das Bundesgericht folgt auch hier der Vorinstanz und hält fest, dass der Prostitutionsvertrag nicht mehr sittenwidrig und der in Frage stehende Vertrag somit gültig zustande gekommen sei.

8 Dazu hält das Bundesgericht in seinen Erwägungen(E. 5.1, 7.1) fest, dass die Inhaltsfreiheit der wichtigste Teil der Vertragsfreiheit gem. Art.19 Abs. 1 OR $^{3}$ sei. Nur ein Vertrag, der einen unmöglichen oder widerrechtlichen Inhalt habe oder gegen die guten Sitten verstosse, sei gem. Art. 20 Abs. 1 OR nichtig.

9 Das Bundesgericht weist sodann auf die zahlreichen Stimmen in der Lehre hin, welche die Sittenwidrigkeit des Prostitutionsvertrags kritisieren und dafür plädieren, diesen als gültig anzuerkennen. Die bisherige Rechtsprechung habe den Prostitutionsvertrag zwar bis anhin als sittenwidrig angesehen, jedoch jeweils lediglich im Sinne eines obiter dictum, ohne genauer auf die Gründe der Sittenwidrigkeit einzugehen. Die Frage, wieso die entgeltliche sexuelle Dienstleistung gegen die gesamtethischen Vorstellungen der Gesellschaft verstosse, sei in den Urteilen offen geblieben. Weiter verweisen die Lausanner Richter:innen auf die Ausführungen in der Lehre, welche die Qualifikation des Prostitutionsvertrags als sittenwidrig im Hinblick auf die heute anerkannten sozialethischen Wertvorstellungen als anachronistisch betrachten. Dieser Wertewandel habe sich auch in der Stellungnahme des Bundesrates aus dem Jahr 2012 zur Interpellation «Privatrechtliche Anerkennung des Prostituiertenlohnes» und im Bericht der Kommission für Rechtsfragen des Ständerates aus dem Jahr 2016 zu der vom Kanton Bern im Jahr 2012 beim Bund eingereichten Standesinitiative «Prostitution ist nicht sittenwidrig» gezeigt. Die Kommission komme in diesem Bericht zum Schluss, dass die Gerichte Verträge über entgeltliche sexuelle Dienstleistungen künftig nicht mehr als sittenwidrig qualifizieren würden und eine ausdrückliche gesetzliche Regelung deshalb überflüssig sei (E. 7.1).

Das Bundesgericht führt weiter aus, dass der Anspruch auf Entschädigung nach erbrachter Leistung der Privatklägerin aus Prostitutionsvertrag strafrechtlich schutzwürdig sei. Das Erwerbseinkommen von Sexarbeiter:innen sei als rechtmässig anerkannt und in verschiedener Hinsicht rechtlich erfasst. Die Sexarbeit sei sodann eine sozialübliche und zulässige Tätigkeit, sofern diese nicht

3 Bundesgesetz betreffend die Ergänzung des Schweizerischen Zivilgesetzbuches (Fünfter Teil: Obligationenrecht) vom 30. März 1911 (OR; SR 220). die Tatbestände der Förderung der Prostitution gem. Art. 195 StGB oder der unzulässigen Ausübung der Prostitution gemäss Art. 199 StGB erfülle. So sei die Sexarbeit als wirtschaftliche Tätigkeit durch die Wirtschaftsfreiheit gemäss Art. $27 \mathrm{BV}^{4}$ geschützt. Weiter unterliege diese der Einkommens- und Vermögenssteuer sowie der AHVBeitragspflicht. Das Bundesgericht kommt deshalb zum Schluss, dass der sexuellen entgeltlichen Dienstleistung sowohl in der Praxis als auch in einem Teil der Rechtsordnung ein Vermögenswert beigemessen werde. Die Qualifizierung der Sexarbeit in anderen Rechtsgebieten sei bei der Beurteilung, ob der Anspruch der Sexarbeiter:in auf Entgelt für die erbrachte sexuelle Dienstleistung schutzwürdig sei, mitzuberücksichtigen. Das Bundesgericht führt sodann aus, dass der Vertrag über die Erbringung einer sexuellen Dienstleistung gegen Entgelt nicht in jeder Hinsicht den der Gesamtrechtsordnung immanenten ethischen Prinzipien und Wertmassstäben widerspreche. Dies gelte auch innerhalb des Zivilrechts. Das Bundesgericht habe in der Vergangenheit etwa den Bordellvertrag sowie den Vertrag zwischen Benutzer:innen eines Telefonanschlusses und Anbieter:innen von erotischen oder pornografischen Dienstleistungen per Telefon als gültig betrachtet. Es könne deshalb nicht an der Sittenwidrigkeit des Prostitutionsvertrags festgehalten werden. Das Bundesgericht kommt aus diesen Gründen zum Schluss, dass beim vorliegenden Sachverhalt «der von der Rechtsordnung offensichtlich nicht missbilligten sexuellen Dienstleistung» ein Vermögenswert zukomme. Es sei schliesslich unvereinbar, «einerseits über das Sexualstrafrecht die Handlungsfreiheit der sich prostituierenden Person zu schützen [...] und andererseits in diesem Bereich im Rahmen des Betrugstatbestandes allein die Interessen des Freiers zu verteidigen und so zu einem unsittlichen Gewerbe noch eine unsittliche Ausbeutung hinzuzufügen» (E. 7.2).

Das Bundesgericht stellt damit fest, dass dem Anspruch 11 der Privatklägerin auf Entschädigung Vermögenswert zukomme und der Tatbestand des Betrugs i.S. v. Art. 146 StGB erfüllt sei.

\section{Anmerkungen}

\section{Lang erwartetes Urteil}

Das Bundesgericht hat mit dem zur Publikation vorgese- 12 henen Urteil die Frage der Sittenwidrigkeit von Prostitutionsverträgen geklärt. Das Urteil war lang erwartet, denn aufgrund der sich veränderten Wertevorstellungen in der Gesellschaft kann ein Vertrag über die Erbringung

4 Bundesverfassung der Schweizerischen Eidgenossenschaft vom 18. April 1999 (BV; SR 101). 
sexueller Dienstleistungen gegen Entgelt, ein Sexarbeitsvertrag oder ein Prostitutionsvertrag5, heute nicht mehr als sittenwidrig i.S. v. Art.19/20 OR angesehen werden. Dem Zustandekommen eines Vertrags über sexuelle Dienstleistungen stehen damit im Grundsatz keine Inhaltsschranken mehr entgegen, wodurch dieser gültig zustande kommt und die Erfüllung des Vertrags verlangt werden kann. ${ }^{6}$ Mit den Worten des Bundesgerichts verstösst ein Prostitutionsvertrag heute nicht mehr «gegen die herrschende Moral, d.h.gegen das allgemeine Anstandsgefühlodergegen die der Gesamtrechtsordnung immanenten ethischen Prinzipien und Wertmassstäbe» ${ }^{7}$ und führt folglich nicht mehr zur Nichtigkeit des Vertrages.

Nachfolgend ist zunächst pro memoria kurz auf die Rechtslage in der Schweiz einzugehen (1). Danach stehen die hier interessierenden Erwägungen des Bundesgerichts im Zentrum (2), und schliesslich sollen die sich weiterhin stellenden rechtlichen Fragen im Zusammenhang mit dem Prostitutionsvertrag behandelt werden (3).

\section{Rechtlicher Kontext}

In der Schweiz besteht seit Inkrafttreten des Strafgesetz buches im Jahr 1942 das sog. regulatorische Regime, bei dem die freiwillige Sexarbeit von volljährigen Personen legal ist. ${ }^{8}$ Prostitution von Minderjährigen sowie Menschenhandel zum Zweck sexueller Ausbeutung sind hingegen gemäss Art. 195 Bst. a, Art. 196 sowie Art.182 StGB verboten, da sie gegen die Menschenwürde und die sexuelle Selbstbestimmung verstossen. ${ }^{9}$ So verbietet Art. 196 StGB die Entgegennahme von sexuellen Diensten von Minderjährigen gegen Entgelt. Nach Art.195 Bst. a StGB macht sich strafbar, wer eine minderjährige Person der Prostitution zuführt oder in der Absicht, daraus Vermögensvorteile zu erlangen, ihre Prostitution fördert.

5 Das Bundesgericht verwendet den Terminus «Prostitutionsvertrag», der negativ behaftet bleibt. Ebenso geläufig ist der Begriff «Sexarbeitsvertrag», der richtigerweise die selbstbestimmte Erwerbstätigkeit in den Vordergrund stellt, aber auch nicht ganz korrekt ist, denn es bleibt ein atypischer Arbeitsvertrag (dazu Rz. 26), begriffsneutraler wäre "vertraglich vereinbarter Sex gegen Entgelt», siehe zum Ganzen: SANDRA HOTZ, Selbstbestimmung im Vertragsrecht, Unter besonderer Berücksichtigung von Verträgen zu «Liebe», Sex und Fortpflanzung, Habil. Zürich, Bern 2017, S. 326 (zit. HOTZ, Selbstbestimmung).

6 Zu den Inhaltsschranken gem. Art.19/20 OR siehe BARBARA MEISE / CLAIRE HUGUENIN, in: Widmer Lüchinger/ Oser (Hrsg.), Basler Kommentar, Obligationenrecht I, 7. Aufl., Basel 2020, Art.19/20 OR N12 ff. (zit. BSK OR I-BEARBEITER:IN).

7 BGE136III474 E.3; BGE132 III 455 E. 4.1; BGE123 II 97 E. 2.

8 BRIGITTE HÜRLIMANN, Prostitution - ihre Regelung im schweizerischen Recht und die Frage der Sittenwidrigkeit, Diss. Freiburg 2004, S.1ff. Die homosexuelle Sexarbeit wurde hingegen erst 1992 legalisiert; MARIA SCHULTHEISS, Das gesellschaftliche Verständnis der sich prostituierenden Person und dessen Abbild im Recht, ex ante 2017, S. 36; vgl. zu den internationalen Rechtsgrundlagen zur Bekämpfung von Trafficking Urteil des EGMR 60561/14 vom 24.Juni 2020 (S.M. gegen Kroatien); Urteil des EGMR 40591/11 vom 9. Februar 2021 (N.C. gegen Türkei).

9 HотZ, Selbstbestimmung (Fn. 5), S. 262 ff., 268 ff.
Primäres Ziel des regulatorischen Regimes ist es, die 15 Selbstbestimmung von Sexarbeiter:innen sicherzustellen. Nach Art.195 Bst. b-d StGB sind Beeinträchtigungen der Handlungsfreiheit verboten. Strafbare Handlungen im Sinne der Bestimmung sind die Zuführung einer Person zur Prostitution unter Ausnützung ihrer Abhängigkeit oder wegen eines Vermögensvorteils (lit.b), die Beeinträchtigung der Handlungsfreiheit der sich prostituierenden Person (lit.c) sowie das Festhalten einer Person in der Prostitution (lit.d). Das geschützte Rechtsgut ist einzig die sexuelle Selbstbestimmung bzw. die Handlungsfreiheit der Sexarbeiter:innen. ${ }^{10}$ Art.195 StGB bestraft folglich nur diejenigen Personen, die sich die Zwangslage der Sexarbeiter:innen zu Nutze machen und eine selbstbestimmte Entscheidung verhindern. ${ }^{11}$ Neben Art. 195 wird gem. Art.199 StGB mit Busse bestraft, «wer den kantonalen Vorschriften über Ort, Zeit oder Art der Ausübung der Prostitution und über die Verhinderung belästigender Begleiterscheinungen zuwiderhandelt». Als ein Fremdkörper im Sexualstrafrecht wird Art.199 StGB etwa angesehen, weil der Schutz von polizeilichen Rechtsgütern der Kompetenz der Kantone und nicht des Bundes unterliegt. ${ }^{12}$ Das heisst, die Kompetenz der Kantone zum Erlass polizeilicher Massnahmen zur Regelung der Sexarbeit besteht unabhängig von Art.199 StGB, womit Letzterer eigentlich «nur» den Verstoss besonders bundesrechtlich sanktioniert. ${ }^{13}$ Klar ist: Die kommunalen und kantonalen Vorschriften dürfen die bundesrechtlich zulässige Sexarbeit, welche unstrittig durch Art. 27 BV geschützt ist, nicht unverhältnismässig behindern. ${ }^{14}$

Strafrechtlich wichtig ist, dass das Sexgewerbebetreffend 16 Akteur:innen, Arbeitsort und -bedingungen eine sehr grosse Heterogenität aufweist, was zu beachten ist. ${ }^{15}$ Freiwillige, selbstbestimmte Sexarbeit ist getrennt von der Bekämpfung von Menschenhandel zum Zweck sexueller Ausbeutung geregelt und rechtlich auch so zu behandeln. Das Urteil des Bundesgerichts ist in dem Sinne ein Schritt in die richtige Richtung.

Neben den erörterten strafrechtlichen Bestimmungen 17 ist des Weiteren festzuhalten, dass Sexarbeit vom Bundesgericht seit dem Jahre 1973 unter dem Titel der Wirt-

10 KATHRIN HEINZL, Prostitution im Schweizer Strafrecht, Diss. Zürich, Zürich et al. 2016, S.125; HOTZ, Selbstbestimmung (Fn. 5), S. 264; HÜRLIMANN (Fn. 8), S. 46f.; BERNHARD ISENRING / MARTIN A. KESSLER, in: Niggli/Wiprächtiger (Hrsg.), Basler Kommentar, Strafrecht, Strafgesetzbuch, Jugendstrafgesetz, 4. Aufl., Basel 2019, Art.195 StGB N2f. (zit. BSK StGB-BEARBEITER:IN); Botschaft vom 26. Juni 1985 über die Änderung des Schweizerischen Strafgesetzbuches und des Militärstrafgesetzes (BBl 1985 II 1009) S.1082 (zit. Botschaft 1985).

11 BSK StGB-ISENRING/KESSLER, Art. 195 StGB N 7.

12 BSK StGB-ISENRING, Art.199 StGB N3a.

13 BSK StGB-ISENRING, Art. 199 StGB N 5.

14 HOTZ, Selbstbestimmung (Fn. 5), S. 265.

15 SCHULTHEISS (Fn. 8), S. 39. 
schaftsfreiheit gemäss Art. 27 BV geschützt ist. ${ }^{16}$ Das Einkommen von Sexarbeiter:innen wird seit Jahrzehnten besteuert, ${ }^{17}$ und es werden Sozialversicherungsabgaben ${ }^{18}$ erhoben. Das Entgelt für sexuelle Dienstleistungen ist pfändbar ${ }^{19}$ und stellt im Haftpflichtrecht eine anerkannte Schadensposition ${ }^{20}$ dar. Anders gewendet: «Der Lohn» von Sexarbeiter:innen wird in diesen Rechtsgebieten gleichbehandelt wie jedes andere Erwerbseinkommen. ${ }^{21}$ Es war und ist somit in der Schweizer Rechtsordnung zulässig, mittels Sexarbeit ein Einkommen (volloder teilweise) zu verdienen. ${ }^{22}$

\section{Praxisänderung zu den "guten Sitten»}

a) Vertraglich vereinbarter Sex gegen ein Entgelt ist nicht sittenwidrig

Nach der bisherigen bundesgerichtlichen Rechtsprechung ${ }^{23}$ verstiess ein Prostitutionsvertrag gegen die «guten Sitten» und war deshalb nichtig. Dies hatte zur Folge, dass Sexarbeiter:innen keinen Anspruch auf das mit dem Kunden vereinbarte Entgelt hatten und ihren Lohn nicht einfordern konnten. ${ }^{24}$

Es steht erstmals die Frage nach der Sittenwidrigkeit eines Prostitutionsvertrages im Zentrum eines Urteils des Bundesgerichts (sog. ratio decidendi). ${ }^{25}$ Bisher ging das Bundesgericht nicht explizit auf die Gründe der Sittenwidrigkeit ein, namentlich nicht im bisherigen «Leitentscheid zur Sittenwidrigkeit» aus dem Jahre $1985 .{ }^{26}$ Auch im Jahr 2011 hielt die strafrechtliche Abteilung des Bundesgerichts in einem obiter dictum fest, dass weiterhin davon

16 BGE 101 Ia 473 E. 2 und E. $2 \mathrm{~b}$ «Le fait que la prostitution puisse être considérée comme contre contraire aux moeurs n'as pas pour effet de priver les personnes qui s'y livrent professionnellement du droit d'invoquer l'article 31 Cst.»; BGE 99 Ia 504; weiter siehe Hinweise HOTZ, Selbstbestimmung (Fn. 5), S.274, Fn.1181.

17 BGE111II295E. 2d; siehe auch Urteil des Bundesgerichts 6B_188/2011 vom 26. Oktober 2011 E. 2.3.

18 BGE107V193 E. 2b.

19 BGE111II 295 E. 2d; BGE 91 IV 69 S. 69f. (einzige Erwägung).

20 BGE111II 295 E. 2e.

21 SEBASTIAN REICHLE / ROMAN SCHISTER, Sittenwidrigkeit des Sexdienstleistungsvertrags?, ex ante 2017, S. 22.

22 нотz, Selbstbestimmung (Fn. 5), S. 274.

23 BGE129III 604 E. 5.3; BGE111 II 295 E. 2e; BGE 101 Ia 473 E. 2b; BGE 91 IV 69 (einzige Erwägung betr. Unsittlichkeit des Gewerbes einer Dirne, welche aber nicht zur Folge habe, dass die Pfändung ausgeschlossen sei); als obiter dicta im Urteil des Bundesgerichts 6B_188/2011 vom 26. Oktober 2011 E. 2.3 und im Urteil des Bundesgerichts 4C.50/2003 vom 5.Juni 2003 E. 5.3.

24 HÜRLIMANN (Fn. 8), S. 29; BSK OR I-MEISE/HUGUENIN, Art. 19/20 OR N38.

25 FRÉDÉRIC KRAUSKOPF/JESSICA KIM SOMMER, Sittenwidrig oder nicht?, Das Berner Prostitutionsgesetz vom 7.Juni 2012 und die Diskussion um den Prostitutionsvertrag, in: Kunz/Weber / Lienhard/ Fargnoli / Kren Kostkiewicz (Hrsg.), Berner Gedanken zum Recht, Festgabe der Rechtswissenschaftlichen Fakultät der Universität Bern für den Schweizerischen Juristentag 2014, Bern 2014, S. 62.

26 BGE111 II 295 E. 2e. auszugehen sei, dass der Vertrag über die sexuelle Dienstleistung gegen Geld sittenwidrig sei. ${ }^{27}$

Im Resultat entspricht die bundesgerichtliche Rechtspre- 20 chung dem vorinstanzlichen Urteil und dem bisher ersten bekannten kantonalen Urteil des Bezirksgerichtes Horgen im Kanton Zürich aus dem Jahr 2013, das den Prostitutionsvertrag als gültig ansieht, da dieser nach heutigen gesellschaftlichen Moralvorstellungen nicht mehr als sittenwidrig anzusehen sei. ${ }^{28}$ Damit entspricht die Rechtsprechung des Bundesgerichts einem wesentlichen Teil der Lehre, welche sich seit längerem für die Gültigkeit eines Prostitutionsvertrags ausspricht. ${ }^{29}$

Schliesslich wird mit diesem Entscheid die bis anhin un- 21 befriedigende Rechtslage (wie unter Ziff. 2 bereits skizziert) insoweit geklärt, als einklagbare Rechte bestehen sollen: Es ist ein Einkommen aus Sexarbeit möglich und dieses wird nicht bloss wie bis anhin verschiedenen Abgaben unterliegen, verfassungsrechtlichen Schutz geniessen und strafrechtlich nicht zu beanstanden sein. ${ }^{30}$ Es besteht nunmehr nicht nur verbesserte Rechtssicherheit in Bezug auf das gültige Zustandekommen eines Vertrages zu sexuellen Dienstleistungen, sondern Sexarbeiter:innen werden in ihren Rechten betreffend sexueller und wirtschaftlicher Selbstbestimmung gestärkt. Das Urteil ist aus all diesen Gründen sehr zu begrüssen.

\section{b) Sexuelle Selbstbestimmung und ihre Nähe zu Straf- und Zivilrecht}

Bemerkenswert ist ferner, dass der Entscheid über den 22 strafrechtlichen Schutz des Einkommens der Sexarbeiter:innen hinausgeht und die strafrechtliche Abteilung des Bundesgerichts den Leitentscheid zur überfälligen zivilrechtlichen Frage der Sittenwidrigkeit von Verträgen

27 Urteil des Bundesgerichts 6B_188/2011 vom 26. Oktober 2011 E. 2.3. 28 Urteil des Bezirksgerichts Horgen FV120047 vom 9. Juli 2013, in: ZR112 (2013) Nr. 85.

29 ANDREAS FURRER/ MARKUS MÜLLER-CHEN, Allgemeiner Teil Obligationenrecht, 3. Aufl., Zürich 2018, S. 175; SANDRA HOTZ, Körper, Arbeit und Selbstbestimmung: Massage, Prostitution, Leihmutterschaft, Zur Kommerzialisierung des Privaten - ein Ausblick, ZSR 2019, S.126ff. (zit. HOTZ, ZSR); HOTZ, Selbstbestimmung(Fn.5), S.286f.; HÜRLIMANN (Fn. 8), S. 178, 222 ff.; CLAIRE HUGUENIN, Obligationenrecht, Allgemeiner und Besonderer Teil, 3. Aufl., Zürich et al. 2019, S.130 f.; KRAUSKOPF/SOMMER (Fn. 25), S. 57 ff.; BSK OR I-MEISE/HUGUENIN, Art.19/20 N38; sinngemäss: OLIVIER GUILLOD / GABRIELLE STEFFEN, in: Thévénoz/Werro (Hrsg.), Code des obligations I: Art.1-529 CO, Commentaire, 2. éd., Bâle 2012, art.19 CO N70. A. M.: PETER GAUCH / WALTER R. SCHLUEP / JÖRG SCHMID / SUSAN EMMENEGGER, Schweizerisches Obligationenrecht Allgemeiner Teil, Band I, 11. Aufl., Zürich et al. 2020, Rn.672; ALFRED KOLLER, Schweizerisches Obligationenrecht Allgemeiner Teil, 4. Aufl., Bern 2017 N.13.177 und 13.195f.; wohl ebenfalls a.M. INGEBORG SCHWENZER/CHRISTIANA FOUNTOULAKIS, Schweizerisches Obligationenrecht Allgemeiner Teil, 8. Aufl., Bern 2020, N32.28.

30 Die Annahme der Sittenwidrigkeit des Prostitutionsvertrags wäre heute "vor dem Hintergrund einer einheitlichen und widerspruchsfreien Rechtsordnung» problematisch (BGE 147 IV 73 E. 5.1. in fine). 
im Allgemeinen und dem Prostitutionsvertrag im Besonderen hervorgebracht hat, und nicht, wie rechtsdogmatisch zu erwarten gewesen wäre, die zweite Zivilrechtskammer.

Das zeigt, dass das Vermögensstrafrecht stark zivilrechtsakzessorisch ist. Ob das Strafrecht als besonders symbolhaltig für gesellschaftliche Veränderungen im Bereich der Sexualität angesehen werden kann oder gar als «Katalysator» für solche Entwicklungen wirkt, wie es bereits die bisherige Entwicklung des materiellen Sexualstrafrechts nahelegen, kann hier offen bleiben. ${ }^{31}$ Auch wenn in casu eine adhäsionsweise Geltendmachung der Zivilforderung nötig war, liegt es sicher auch daran, dass eine Strafverfolgung von Amtes wegen erfolgt, denn Sexarbeiter:innen werden sich regelmässig keinen Zivilprozess leisten können, um ihr Entgelt einzufordern. Hinzu kommt, dass bei Querelen im Sexgewerbe aufgrund der besonderen Vulnerabilität der Akteur:innen ${ }^{32}$ und der Stigmatisierung der Involvierten weniger der Gang zu den Behörden gesucht wird. ${ }^{33}$ Die Zahlen sprechen insoweit für sich: Es gibt kaum Gerichtsentscheide, trotz vieler kantonaler Prostitutionsgesetze (dazu gleich unter Ziff. 3) und jährlich 4000 bis 8000 Personen, die im Erotikbereich tätig sind, ${ }^{34}$ wobei andere Schätzungen sehr viel höher liegen und von 13'ooo bis 20’ooo Personen ausgehen. ${ }^{35}$ - Insoweit handelt es sich vorliegend nicht zufällig um einen Entscheid der strafrechtlichen Kammer und ein ausstehendes Entgelt von CHF 2000, denn das ist betragsmässig überdurchschnittlich. Die Privatklägerin durfte zudem von der Zahlungsfähigkeit des Kunden ausgehen und wurde über dessen Zahlungswillen getäuscht.

31 Seit der Kodifizierung des eidgenössischen Strafrechts im Jahre 1942 ist selbstbestimmte Erwachsenenprostitution zugelassen, wobei die homosexuelle Prostitution noch bis 1992 verboten blieb. Homosexualität für sich genommen war aber 1942 nicht mehr strafbar, wobei das Schutzalter für gleichgeschlechtliche Sexualität ungleich höher blieb. Mit der Sexualstrafrechtsrevision von 1992 wurde das überkommene «Sittlichkeitsstrafrecht» aufgegeben, und es wurden neu Straftatbestände zum Schutz der sexuellen Selbstbestimmung eingeführt. Seit Inkrafttreten des Opferhilfegesetzes im Jahr 1993 wurde die rechtliche Stellung und Unterstützung der Opfer von Gewaltdelikten stark verbessert: Botschaft 1985(Fn. 9), S. 1064. ERASMUS WALSER, Homosexualität im Recht, Historisches Lexikon der Schweiz.

32 Gem. Schätzungen von NGOs sind ca. 75 Prozent der Frauen im Sexgewerbe Migrantinnen, s. dazu Fachstelle für Frauenhandel und Frauenmigration, Factsheet Sexarbeit, Mai 2017 (zit. FIZ, Factsheet).

33 HотZ, Selbstbestimmung (Fn. 5), S. 278.

34 Da sich etwa mehrere Sexarbeiter:innen eine Arbeitsstelle über ein Jahr hinweg teilen: LORENZ BIBERSTEIN / MARTIN KILLIAS, Erotikbetriebe als Einfallstor für Menschenhandel? Eine Studie zu Ausmass und Struktur des Sexarbeitsmarktes in der Schweiz, Lenzburg 2015, S. 12. Und umgekehrt nimmt fast jeder fünfte Mann in der Schweiz zwischen 20 bis 65Jahren einmal proJahr eine entgeltliche sexuelle Dienstleistung entgegen: Aids-Hilfe Schweiz, Jahresbericht 2014, Programm Sex Work, 2015, S. 22.

35 GÉRALDINE BUGNON/MILENA CHIMIENTI/LAURA CHIQUET, SeXmarkt in der Schweiz, Teil 3: Mapping, contrôle et promotion de la santé dans le marché du sexe en Suisse, Genève 2009.
Es liesse sich an dieser Stelle freilich einwenden, dass es 24 nur deshalb kaum Gerichtsentscheide gebe, weil es in dieser Branche üblich sei, auf Vorauszahlung zu arbeiten; doch dem kann hier nicht gefolgt werden. Dies muss als eine gängige Annahme hinterfragt werden. ${ }^{36}$ Mit dieser «Annahme» hängt auch der Vorbehalt des Bundesgerichts zur Arglist zusammen (E. 4.2): Wäre die Vorausbezahlung in der Berufspraxis üblich, wovon aber hier nicht ausgegangen wird, könnte allenfalls das Tatbestandselement der Arglist fehlen. Es dürfte jedenfalls nur dann von einer Vorauszahlungpflicht ausgegangen werden, wenn rechtliche Grundlagen geschaffen würden, denn alles andere hiesse, dass von den Personen in diesem Gewerbe ungleich Personen in anderer Gewerben erwartet würde, dass sie sich defacto entsprechend verhielten und namentlich von ihnen erwartet würde, ungleich anderen Personen, dass sie sich entsprechenden Drucksituationen (z.B. Feilschen um Entgelt) widersetzen können.

\section{c) Vorstellungen zu Sex, Fortpflanzung und Familie bleiben nicht «beständig»}

Die Erwägungen des Bundesgerichts zu den "guten Sit- 25 ten" gehen nach der hier vertretenen Ansicht aber weit über die Frage der Sittenwidrigkeit des Prostitutionsvertrages hinaus und sind von allgemeiner Bedeutung für das Vertragsrecht: Denn das höchste Gericht hält fest, dass nur Verträge «mit eindeutig schwerwiegenden Verstössen gegen die öffentliche Ordnung oder anerkannte und im Wandel der Zeit beständige Moralvorstellungen sittenwidrig sein können» und die "guten Sitten» nur als ein «Notventil» verstanden werden dürfen. Hochinteressant ist der Satzteil «im Wandel der Zeit beständige Moralvorstellungen sein können» (E. 7.1), denn das heisst nichts anderes, als dass nur das, was über die Zeit unveränderlich konstant als allgemeingültig stossend angesehen werden muss, auch «sittenwidrig» sei. Verwiesen wird dabei auf ein aussagekräftiges Urteil der zweiten Zivilrechtskammer des Bundesgerichts ${ }^{37}$ (unter Alt-Bundesrichter Walter), das Waffengeschäfte an die Republik Kroatien betrifft und in dem auf den Grundsatz des Gewaltverzichts der UN-Charta referenziert wird, welcher in den meisten nationalen Rechtsordnungen und insbesondere auch in der schweizerischen Kriegsmaterialpolitik seinen Niederschlag gefunden habe, womit es sich um einen ethisch höchststehenden universellen Wert handle, «der allen Kulturstaaten gemeinsam ist».38 Das legt umgekehrt nahe, dass im Hinblick auf gesellschaftliche Vorstellungen von Sex, Fortpflanzung oder Lebens- sowie Familienformen, über die unbestrittenermassen im Wandel der Zeit

\footnotetext{
36 Z.B. REICHLE/SCHISTER (Fn. 21) S. 22; a. M. HOTZ, Selbstbestimmung (Fn. 5), Anhang I; diese Annahme bildete nämlich den Ausgangspunkt dieser Arbeit und die zugegebenermassen limitierten Erhebungen in der Branche haben damals ergeben, dass dies nicht der Fall ist.

37 Urteil des Bundesgerichts 4C.172/2000 vom 28. März 2001 E. 5e. 38 Urteil des Bundesgerichts 4C.172/2000 vom 28. März 2001 E. 5 f.
} 
verschiedene Moralvorstellungen bestanden, bestehen und bestehen werden, keine Sittenwidrigkeit mehr anzunehmen ist. Ausgenommen, es bestehe ein schwerwiegender Verstoss gegen die öffentliche Ordnung.

\section{Weitere Massnahmen gegen Stigmatisierung und Diskriminierung bleiben nötig}

\section{a) Ein Vertrag mit besonderen Schutzpflichten} bringung sexueller Dienstleistungen gegen Entgelt bleibt aber auch nach diesem Urteil unklar, da diese im Gesetz nicht speziell geregelt sind. ${ }^{39}$

Es kommen somit die allgemeinen und besonderen Bestimmungen des Obligationenrechts zur Anwendung. Der Bundesrat vertritt in seinem Bericht aus dem Jahr 2015 die Ansicht, dass der Prostitutionsvertrag unter das Auftragsrecht i. S. v. Art. 394 ff. OR fällt. ${ }^{40}$ Demnach können die Parteien gem. Art.404 OR den Vertrag jederzeit beenden. Kund:innen können Sexarbeiter:innen folglich nicht zur Erbringung der sexuellen Dienstleistung gegen ihren Willen zwingen. ${ }^{41}$ Neben der jederzeitig möglichen Beendigung gemäss Art. 404 OR kann ein:e Sexarbeiter:in auch gestützt auf Art. 27 f. $\mathrm{ZGB}^{42}$ die sexuelle Dienstleistung verweigern. ${ }^{43}$ Generell stellt sich auch die Frage, wie sich ein Prostitutionsvertrag (unabhängig von der Qualifikation) zu den Bestimmungen der Vertragsverletzung verhält. Aufgrund des Persönlichkeitsrechts gemäss Art. 27f. ZGB ist klar, dass grundsätzlich keine Erfüllung von sexuellen Dienstleistungen geltend gemacht werden kann; Nicht- und Schlechterfüllungsansprüche sind allerhöchstens aufgrund beweisbarer Vereinbarungen sowie im Rahmen von Schadenersatzansprüchen denkbar. Denkbar ist deshalb die Konzeption als einseitig verbindlicher Vertrag wie nach deutschem Prostitutionsgesetz (\$1 Satz1 ProstG $\left.{ }^{44}\right) .{ }^{45}$ Nach dieser Bestimmung haben Sexarbeiter:innen einen Anspruch auf Zahlung des vereinbarten Entgelts nach Erbringung ihrer Leistung, die Kund:innen hingegen haben gestützt auf Vertrag keinen Anspruch auf Erbringung der sexuellen Dienstleistung.

39 нотZ, Selbstbestimmung (Fn. 5), S. 273.

40 Bundesrat, Prostitution und Menschenhandel zum Zweck der sexuellen Ausbeutung, Bericht des Bundesrates in Erfüllung der Postulate 12.4162 Streiff-Feller, 13.3332 Caroni, 13.4033 Feri und 13.4045 Fehr vom 5. Juni 2015 (zit. Bericht Bundesrat); so auch REICHLE/SCHISTER (Fn. 21), S. 29. Siehe auch HÜRLIMANN (Fn. 8), S.162 f. und $221 \mathrm{ff}$.

41 Siehe zum Ganzen HürLIMANN (Fn. 8), S. $221 \mathrm{ff}$.

42 Schweizerisches Zivilgesetzbuch vom 10. Dezember 1907 (ZGB; SR 210).

43 REICHLE/SCHISTER (Fn. 21), S. 25; für Art. 27 ZGB: KRAUSKOPF/SOMMER (Fn. 25), S. $70 \mathrm{f}$.

44 Gesetz zur Regelung der Rechtsverhältnisse der Prostituierten vom 20. Dezember 2001 (Prostitutionsgesetz, ProstG; FNA 402-39).

45 HOTZ, Selbstbestimmung (Fn. 5), S. 289 ff., 296; hier wird das Modell zweiseitiger Vertrag mit einseitig durchsetzbarem Anspruch bevorzugt (S.328).
Eine zusätzliche Unklarheit ist, inwiefern Sexarbeiter:in- 28 nen rechtlich und tatsächlich als unselbstständig Erwerbstätige gem. Art. $319 \mathrm{ff.} \mathrm{OR} \mathrm{arbeiten} \mathrm{können.}{ }^{46}$ Ein Grund für die Unsicherheit liegt zum einen im Auslegungsspielraum von Art. 195 lit.b-d StGB, welcher für Arbeitgeber:innen die Gefahr birgt, sich strafbar zu machen. Insbesondere lit. c - nach welchem sich strafbar macht, wer die Handlungsfreiheit einer Person, die Prostitution betreibt, dadurch beeinträchtigt, dass er sie bei dieser Tätigkeit überwacht oder Ort, Zeit, Ausmass oder andere Umstände der Prostitution bestimmt - ist in diesem Zusammenhang von Bedeutung. Das Bundesgericht hat bisher keine klaren Kriterien für die Erfüllung des Tatbestandes festgelegt. Aufgrund der unklaren Rechtslage verzichtet ein Betrieb lieber auf den Abschluss eines Arbeitsvertrags mit Sexarbeiter:innen. ${ }^{47} \mathrm{Hinzu}$ kommt, dass das Weisungsrecht der Arbeitgeber:innen gemäss Art. 321d OR dem Persönlichkeitsrecht von Sexarbeiter:innen nach Art. 27 ZGB entgegen steht: Geltend gemacht wird etwa, dass das Weisungsrecht zu einem verstärkten Machtgefälle führen könne ${ }^{48}$ und die Erbringung einer sexuellen Dienstleistung klarerweise dem arbeitsrechtlichen Weisungsrecht entzogen werde. ${ }^{49}$ Trotz dieser rechtlichen Hindernisse kann es möglich sein, einen Arbeitsvertrag mit Sexarbeiter:innen abzuschliessen. ${ }^{50}$ Für die explizite Zulässigkeit einer unselbstständigen Tätigkeit spricht sich beispielsweise die Regelung im Prostitutionsgewerbegesetz des Kantons Bern aus. ${ }^{51}$ Auch weitere Kantone anerkennen die unselbstständige Erwerbstätigkeit und stellen Musterverträge für Sexarbeiter:innen zur Verfügung. ${ }^{52}$ Die Vorteile eines Arbeitsvertrags liegen auf der Hand: Dank umfassender Versicherung profitieren sie von der obligatorischen Unfallversicherung, von Lohnfortzahlungen bei Krankheit oder Unfall und von der Arbeitslosenversicherung. Eine weitere Möglichkeit ist es, Verträge über die Erbringung einer sexuellen Dienstleistung als Innominatverträge $\mathrm{zu}$ qualifizieren. ${ }^{53}$

46 Bericht Bundesrat (Fn. 41), S.125ff.; SUSANNE BERTSCHI, Sexarbeit tabuisiert - zum Nachteil der Frauen, Bulletin Nr. 7 des NFP, Bern 2003, S.31f.; HÜRLIMANN (Fn. 8), S.130; SCHULTHEISS (Fn. 8), S.36.

47 HÜRLIMANN (Fn. 8), S.130 ff.; HOTZ, Selbstbestimmung (Fn. 5), Anhang I.

48 Bundesamt für Migration (BFM), Bericht der nationalen Expertengruppe, «Schutzmassnahmen für Frauen im Erotikgewerbe» (zit. Bericht Hilber), Bern 2014, S. 18.

49 THOMAS GEISER / THOMAS OECHSLE, Qualifikation von Verträgen mit Frauen im Erotikbereich, Kurzgutachten im Auftrag des Vereins PROKORE, St. Gallen 2016, S. 9 f.

50 HÜRLIMANN (Fn. 8), S.130.

51 Art. 2 Abs. 3 Gesetz über das Prostitutionsgewerbe vom 7.Juni 2016 des Kantons Bern (PGG BE; BSG 935.90); siehe weitere Beispiele HOTZ, Selbstbestimmung (Fn. 5), S. $275 \mathrm{ff}$.

52 Z.B. Kanton St. Gallen.

53 Bericht Hilber (Fn.48), S.17f.; Bericht Bundesrat (Fn. 40), S. 20f.; ausführlich HÜRLIMANN (Fn. 8), S. $228 \mathrm{ff}$. 
Schweiz eine explizite gesetzliche Regelung für Verträge über sexuelle Handlungen gegen Entgelt. Denn es handelt sich nach der hier vertretenen Ansicht um einen besonderen Typus von Dienstleistungsvertrag, der sehr persönliche, intime und körperliche Dienstleistungen der dienstleistenden Person zum Inhalt hat, und der deshalb auch besondere Schutz- und Fürsorgepflichten vorsehen muss, wie beispielsweise Informationspflichten und Widerrufsrechte. Ein solcher Vertrag müsste aber bezüglich sämtlicher sozialversicherungsrechtlicher Aspekte gleich dem Arbeitsrecht behandelt werden. ${ }^{54}$

In Deutschland gilt etwa neben dem bereits erwähnten Prostitutionsgesetz aus dem Jahre 2001, das den gesetzlichen Anspruch auf Entgelt festlegt, seit dem 1.Juli 2017 auch das Prostituiertenschutzgesetz. ${ }^{55}$ In diesem Gesetz wird u.a. klargestellt, dass Prostituierte selbstständig oder im Rahmen eines Beschäftigungsverhältnisses tätig sein können. Sind Prostituierte angestellt, haben sie die gleichen Rechte und Pflichten wie andere Arbeitnehmer:innen. Sie werden demnach zur Sozialversicherung angemeldet und zahlen in die Kranken-, Arbeitslosen-, Renten-, Pflege- und Unfallversicherung ein. Zugleich ist geklärt, dass in Bezug auf das Erbringen von sexuellen Dienstleistungen kein Weisungsrecht seitens der Betreiber:innen besteht ( $\$ 3$ ProstG, §26 Abs. 2 ProstSchG). Gewerbetreiber:innen müssen ihren Betrieb auch behördlich genehmigen lassen ( $\$ 12$ ProstSchG). Möchte jemand im Prostitutionsgewerbe tätig werden, kann sie oder er sich von den Betreibenden das Betriebskonzept vorlegen lassen (§26 Abs. 5 ProstSchG). Dadurch können Sexarbeiter:innen etwa vorab in Erfahrung bringen, ob der Betrieb die gesetzlich vorgeschriebenen Mindestanforderungen einhält. - Das bedingt allerdings nach ProstSchG, dass Sexarbeiter:innen verpflichtet sind, sich anzumelden ( $\$ 3$ ProstSchG) und ihre Anmeldebescheinigung mit sich führen müssen ( $\$ 4$, $\$ 5$ Abs. 7 ProstSchG), was wiederum stigmatisierend und rechtlich problematisch für die Betroffenen sein kann. ${ }^{56}$

54 Ein Vorschlag siehe HoTz, Selbstbestimmung (Fn. 5), S. $326 \mathrm{ff}$

55 Gesetz zur Regulierung des Prostitutionsgewerbes sowie zum Schutz von in der Prostitution tätigen Personen vom 21. Oktober 2016 (Prostituiertenschutzgesetz, ProstSchG; FNA 402-42).

56 Zum Ganzen siehe ULRIKE LEMBKE, Zwischen Würde der Frau, reduziertem Liberalismus und Gleichberechtigung der Geschlechter, Feministische Diskurse um Prostitution/Sexarbeit, in: Baer/Sacksofsky (Hrsg.), Autonomie im Recht - Geschlechtertheoretisch vermessen, Baden-Baden 2018, S. $275 \mathrm{ff}$.; HOTZ, Selbstbestimmung (Fn. 5), S. 254 .

\section{b) Kantonale und kommunale Hindernisse - das zeigt sich besonders zu Covid-19-Zeiten}

Dem entspricht insoweit das kantonale und kommunale 31 Recht, das oftmals Bewilligungs- und/oder Meldepflichten vorsieht. ${ }^{57}$ Diese mögen zwar als Schutz für Sexarbeiter:innen gedacht und für deren Information sowie zur Bekämpfung des Menschenhandels formuliert sein, in der Praxis verhindern sie aber oftmals auch, dass Sexarbeiter:innen selbstbestimmt, legal und unter sicheren Bedingungen arbeiten, und sollten hinterfragt werden. ${ }^{58}$

Die föderale Struktur im Bereich des Polizeirechts und 32 die damit einhergehende heteronome gesetzliche Regelung von Sexarbeit bringt zusätzliche Unsicherheiten mit sich und es stellt sich regelmässig die Frage, ob die kantonalen und kommunalen Normen die grundrechtlich geschützte Sexarbeit unverhältnismässig einschränken. ${ }^{59}$ Auch die Praxis im Bau- und Raumplanungsrecht perpetuiert Stigmata gegenüber Sexarbeiter:innen, indem ideelle Immissionen im Zusammenhang mit dem Sexgewerbe in der Regel unhinterfragt als gegeben angesehen werden. ${ }^{60}$ Die Sexarbeit sollte beispielweise nicht an den Stadtrand gedrängt werden, da Sexarbeiter:innen dadurch weniger sicher sind und «unsichtbar» gemacht werden.

Jüngste Beispiele für solche föderalen Hindernisse, wel- 33 che der Selbstbestimmung zuwiderlaufen und die noch bestehende Stigmatisierung und Diskriminierung immer wieder verfestigen, sind die kantonalen Sexarbeitsverbote zu Covid-19-Zeiten. Während Prostitution unter Einhaltung von Schutzmassnahmen auf Bundesebene nicht explizit verboten ist (Ausnahme: Clubs), können die Kantone zusätzliche Massnahmen festlegen, sofern es die epidemiologische Lage erfordert: $\$ 5$ Abs. 1 der Verordnung über Massnahmen zur Bekämpfung der Covid-19-Epidemie des Regierungsrates des Kantons Zürich vom 24. August 2020 lautet: «Prostitution ist verboten. Bordell- und Erotikbetriebe, Cabarets und ähnliche Lokale müssen geschlossen bleiben». ${ }^{61}$ Es bestehen zum einen Ungleichbehandlungen zu anderen Gewerben wie dem Massageoder dem Kosmetikgewerbe, wo ebenfalls sehr naher Körperkontakt besteht. Zum anderen haben einzelne Kantone die Prostitution in Covid-19-Zeiten verboten (Kantone Aargau, Basel-Stadt, Nidwalden, Zürich), während

57 HOTZ, Selbstbestimmung (Fn. 5), S. $275 \mathrm{ff}$

58 FIZ, Factsheet (Fn. 32).

59 SCHUlThEISS (Fn. 8), S.37; vgl. Urteildes Bundesgerichts 2C_106/2015 vom 26. Juni 2015 E. 4.1.; BGE137 I167 E. 3.1; BGE101 Ia 473 E. 4 b.

60 SCHULTHEISS (Fn. 8), S. 37.

61 Kanton Zürich, LS 818.18; die Verordnung galt vom 24. August 2020 bis zum 31. Mai 2021 (ABl2021-04-16); Art. 5, 8 Covid-19-Verordnung vom 19. Juni 2020 (SR 818.101.26). 
sie in anderen erlaubt blieb (Kantone Bern, Graubünden, Wallis), was etwa dazu führte, dass die Sexarbeiter:innen bei ihrer Arbeitsausführung auf andere Kantone ausweichen mussten. ${ }^{62}$ Denn nur so konnten sie ein Einkommen erzielen, da nur die wenigsten von ihnen Anspruch auf Kurzarbeitsentschädigung oder Sozialhilfe haben. Es bestehen keine nationalen Vorgaben zu diesen Verboten, die offiziell aufgrund von Ansteckungsgefahr und einer erschwerten Rückverfolgbarkeit von Übertragungsketten erlassen worden sind. Solche Verbote (ob föderaler oder nationaler Natur) illustrieren einerseits, wo Regelungslücken und Machtgefälle liegen und andererseits, dass grundsätzlich zu wenig darüber nachgedacht wird, welche Konsequenzen rechtliche Massnahmen für die unmittelbar betroffenen Sexarbeiter:innen haben. Aufgrund der finanziellen Einbussen müssen Sexarbeiter:innen nämlich trotz Verboten weiterarbeiten, wodurch der Gesundheitsprävention wiederum kein Dienst getan wird: Durch andauernde Marginalisierung, Stigmatisierung und Kriminalisierung sind weder Prävention noch Behandlung einfach.63

\section{Sexuelle Selbstbestimmung und Schutz}

Gültigkeit eines selbstbestimmten Vertrages zu Sex ist aber nicht nur Ausdruck von Selbstbestimmung und ein Abschied von überholten Sittlichkeitsvorstellungen, sondern ein wirksames Schutzinstrument. Wird vor Vertragsschluss nämlich gesprochen und verhandelt - so

62 In der Stadt Bern waren in dieser Zeit rund doppelt so viele Sexarbeiter:innen gemeldet, was zu grosser Platzknappheit und zu unwürdigen Arbeitsbedingungen führte, vgl. MARTINA KOCH / CHRISTIAN LICHTI, «So prekär ist die Lage der Sexarbeiter:innen», Sendung SRF vom 29. Januar 2021. Siehe auch ProCoRe, Expertinnenbericht, Covid-19 Massnahmen und Sexarbeit in der Schweiz: Kantonale Verbote, Gesundheitsrisiken, Gewalt und Ausbeutung vom 15. März 2021, S. 3: Daten zur Verbreitung von Covid-19 unter Sexarbeiter:innen liegen nicht vor. ProCoRe-Fachstellen verzeichnen keine überdurchschnittlich hohen Raten von Covid-19-Erkrankungen unter Sexarbeiter:innen.

63 International Committee on the Rights of Sex Workers in Europe (ICRSE), Sex workers on the frontline, The role of sex workers rights groups in providing support during Covid-19 crisis in Europe, March 2021, S. 5 ff., $8 \mathrm{ff}$. wie es einem Vertrag eigen ist und sein sollte - statt diesen heimlich, im Dunkeln oder am Strassenrand auf die Schnelle abzuschliessen, bewirkt das nicht nur mehr Reflexion und Abwesenheit von Missverständnissen, sondern Consent und (eher) Abwesenheit von Gewalt als Bedingung für einen gültigen Vertrag. Wir verstehen aus diesem Grunde Verträge zu Sex nicht nur als Ausdruck von Selbstbestimmung, sondern auch als Teil des (Eigen-) Schutzes für die Beteiligten. Entsprechend sollte ein Vertrag zu Sex wie auch ein solcher zu anderen körpernahen und nahegehenden intimen Inhalten wie Fortpflanzung, Personensorge (Pflege, Betreuung und Behandlung) oder zu Beziehungsformen und Elternschaft als solche gedacht werden, ${ }^{64}$ denn sie haben aufgrund der Vertragsnatur das Potenzial, sowohl die Selbstbestimmung als auch den Schutz zu stärken. Gespräche und Verhandlungen sind Ausdruck von Selbstbestimmung und damit wichtige erste Schritte zum Schutz. ${ }^{65}$ Das soll freilich nicht heissen, dass ein gültiger Vertrag ein Allheilmittel ist, Verträge nicht gebrochen werden und nicht andere regulatorische Regime weiterhin zu prüfen sind. ${ }^{66}$ Die Festschreibung der Opferrolle durch Verbote dürfte den verschiedenen Lebensrealitäten indes nicht gerecht werden und spricht den Sexarbeiter:innen ihr Selbstbestimmungsrecht ab. ${ }^{67} \mathrm{Im}$ aktuellen Umgang mit Prostitution in Covid-19-Zeiten wird besonders deutlich, dass Sexarbeiter:innen nach wie vor gesellschaftlicher Diskriminierung und Stigmatisierung ausgesetzt sind und ihre rechtliche Stellung sowie finanzielle Absicherung mangelhaft sind. 68

64 HOTZ, Selbstbestimmung (Fn. 5), S. 281, 328f.

65 Nichts anderes zeigt sich unserer Meinung nach in der aktuellen Diskussion um die Revision des materiellen Sexualstrafrechts, Bericht der Kommission für Rechtsfragen des Ständerates vom 28. Januar 2021, Strafrahmenharmonisierung und Anpassung des Nebenstrafrechts an das neue Sanktionenrecht (18.043), Vorlage 3: Bundesgesetz über eine Revision des Sexualstrafrechts, S. 22 ff.; die Vernehmlassungsfrist endete am 10. Mai 2021. Bestünde eine Selbstverständlichkeit, über einvernehmlichen Sex zu sprechen und zu verhandeln, so wäre klar, dass im Straf-, Personen- und Vertragsrecht sexuelle Handlungen - ob entgeltlich oder nicht - gleichermassen auf einer informierten Einwilligung oder gegenseitigen Willenserklärungen beruhen.

66 Es wird bekanntlich in Europa mit Sexarbeit rechtlich sehr unterschiedlich umgegangen: Das seit 1999 in Schweden geltende «Sexkaufverbot» will die Abolition von Sexarbeit und kriminalisiert ausschliesslich die Person, die für sexuelle Dienstleistungen bezahlt, nicht jedoch die Person, die sexuelle Leistungen anbietet. Das sog. schwedische Modell unterliegt verschiedenen Kritikpunkten: Einerseits wird die zugrundeliegende Ideologie und andererseits die praktische Umsetzung und Wirkung angezweifelt: siehe Hотz, Selbstbestimmung (Fn. 5), S. 252 ff.; bspw. Council of Europe, Parliamentary Assembly, Resolution 1983 (2014), Prostitution, trafficking and modern slavery in Europe, Ziff. 12.1.1.; SUSANNE DODILLET / PETRA ÖSTERGREN, Das Schwedische Sexkaufverbot, Beanspruchte Erfolge und dokumentierte Effekte, in: Greif(Hrsg.), SexWork(s), verbieten - erlauben - schützen?, Linzer Schriften zur Frauenforschung, Linz 2012, S. 69 ff., 71, 79f., 83 ff., 85 .

67 SCHULTHEISS (Fn. 8), S. 38.

68 FIZ, Factsheet (Fn. 32). 
Es reicht nicht, umzusetzen, dass die Erbringung von sexuellen Dienstleistungen der Selbstbestimmung zugänglich, legal und nicht mehr sittenwidrig ist. Vielmehr müssen sämtliche staatlichen Vorschriften wertungskohärent ausgerichtet sein, und die moralischen Sittlichkeitsvorstellungen dürfen nicht durch die Hintertüre wieder aufgelebt werden lassen. Dazu braucht es namentlich sichere Arbeitsbedingungen, ${ }^{69}$ aber generell einen

69 HOTZ, ZSR (Fn. 29), S.112. verlässlichen rechtlichen Rahmen, der sowohl einen niederschwelligen Zugang zu Beratungsstellen und Gesundheitsversorgung ermöglicht, wie auch den Einbezug von Sexarbeiter:innen im öffentlichen Diskurs stärkt und darüber hinaus legale und sichere Migrationsmöglichkeiten bedenkt. ${ }^{70}$

70 BARBARA KAVEMANN / ELFRIEDE STEFFAN, Zehn Jahre Prostitutionsgesetz und die Kontroverse um die Auswirkungen, APuZ 2013, S.14. 